\title{
Auditory recognition without identification
}

\author{
Anne M. Cleary \\ Colorado State University, Fort Collins, Colorado \\ MORIAH M. WinfIELD \\ Case Western Reserve University, Cleveland, Ohio \\ AND \\ BogDAN KostiC \\ Colorado State University, Fort Collins, Colorado
}

\begin{abstract}
When visual recognition test items are unidentifiable - through fragmentation, for example - participants can discriminate between unidentifiable items that were presented recently and those that were not. The present study extends this recognition without identification phenomenon to the auditory modality. In several experiments, participants listened to words and were then presented with spoken recognition test items that were embedded in white noise. Participants attempted to identify each spoken word through the white noise, then rated the likelihood that the word was studied. Auditory recognition without identification was found: Participants discriminated between studied and unstudied words in the absence of an ability to identify them through white noise, even when the voice changed from male to female and when the study list was presented visually. The effect was also found when identification was hindered through the isolation of particular phonemes, suggesting that phoneme information may be present in memory traces for recently spoken words.
\end{abstract}

Recognition without identification is the finding that when identification of recognition test items is hindered, participants can discriminate between unidentified studied and unidentified unstudied items. This effect was first shown with word fragments (Cleary \& Greene, 2000; Peynircioğlu, 1990). When participants view a word (e.g., RAINDROP) and then receive a test list, containing word fragments (e.g., R__DR__P), in which half of the fragments correspond to studied words and half to unstudied words, participants can discriminate between unidentifiable fragments of studied words and unidentifiable fragments of unstudied words. In the procedure, for every fragment presented on the test, participants are first asked to identify the word from the fragment. Then, whether or not they are able to do so, they are asked to rate the likelihood that the fragment came from a word on the study list. It has been shown repeatedly with this paradigm that participants give significantly higher recognition ratings to unidentified studied fragments than to unidentified unstudied fragments (Cleary, 2002; Cleary \& Greene, 2000, 2001; Lloyd, Westerman, \& Miller, 2007; Peynircioğlu, 1990).

The paradigm used to elicit recognition without identification presents a potential methodological means of investigating the types of isolable features that can give rise to recognition memory in standard list-learning paradigms. Memory researchers have long assumed that memory traces for studied events exist as compilations of separable features. For example, Tulving and Bower (1974) state:

A rather general and atheoretical conception of the memory trace of an event regards it as a collection of features or a bundle of information. This view has been proposed and elaborated by many writers (e.g., Anisfeld \& Knapp, 1968; Bower, 1967; Bregman \& Chambers, 1966; Underwood, 1969; Wickens, 1970) and is now generally accepted as one of the basic pretheoretical assumptions. (p. 269)

This pretheoretical assumption is evident in many formal models of memory (e.g., Nairne, 1990, 2001), including recognition memory (e.g., Hintzman, 1988). For example, in the global matching models of recognition (see Clark \& Gronlund, 1996, for a review), memory traces are stored as sequences of features, and recognition decisions are based on the global match between the features present in the test probe and all of the features that have been stored in memory from the encoding phase.

Because the paradigm used to elicit recognition without identification involves hindering identification through the isolation of particular item features, it can be viewed as a useful methodological means of investigating the types of isolable item features that can give rise to recognition. Note that with the aforementioned studies involving rec-

A. M. Cleary, anne.cleary@colostate.edu 
ognition without identification of word fragments, the isolated features on which recognition is based are particular letters in particular locations (e.g., R__DR__P). Presumably, when a fragment's corresponding word cannot be identified, the recognition decision must be based on the features available in the fragment. In this case, those features would be particular letters in particular locations.

The recognition without identification paradigm can be used to probe the features involved in recognition memory in the following way: Identification of the recognition test items can be hindered through the isolation of particular types of item features at test, and the specific types of features that can give rise to recognition without identification can then be examined. In fact, Cleary and colleagues have used the recognition without identification paradigm for this purpose. For example, Cleary, Langley, and Seiler (2004) used the paradigm to investigate some types of pictorial features that can give rise to recognition memory for pictorial stimuli. They presented participants with study lists of black-and-white line drawings. After each study list, participants were presented with a test list containing fragments of line drawings. Following the example of Biederman (1987), two types of picture fragments were used: fragments containing information about the component parts of their corresponding pictures (geon fragments), and fragments containing only the middle segments of lines (nongeon fragments). For each picture fragment presented at test, participants were first asked to identify the picture from the fragment; then, whether or not they could identify the picture, they were asked to rate the likelihood that the picture fragment corresponded to a studied picture. Recognition without picture identification was found, but only for geon fragments; no recognition without picture identification effect occurred when nongeon fragments were used. This finding suggests that a type of feature that can be used in the recognition of picture stimuli may be the component parts of a picture, or the picture's geon information.

Cleary (2004) used the recognition without identification paradigm to isolate particular types of word features that can give rise to recognition when word stimuli are used. Participants studied lists of words, each of which was followed by a test list containing cues. The test cues either resembled studied words on a particular feature dimension, or they did not. The feature dimensions that were examined were orthography, phonology, and meaning. To examine orthographic features, cues resembling studied items orthographically were used (e.g., CHEETOHS might be the cue for the studied word CHEETAH). To examine phonological features, cues rhyming with studied words but not looking like them were used (e.g., LAUGHED might be used as a cue for the studied word RAFT). To examine the role of meaning, cues relating to studied words semantically were used (e.g., JAGUAR might serve as the cue for the studied word CHEETAH). Recognition without identification was shown in all of these cases. Specifically, participants gave higher recognition ratings to cues resembling studied words than to cues not resembling studied words, in the absence of an ability to identify the studied words that the cues resembled.
Thus far, the recognition without identification paradigm has been used only to isolate features visually on the recognition test. The present study explores the use of the recognition without identification paradigm for isolating auditory features and examining their role in recognition. To this end, the present study attempted to find recognition without identification in the auditory realm. To prevent identification auditorily, analogs to methods of visually preventing identification were used here; specifically, the auditory analog to visually masking rapidly flashed words (e.g., Cleary \& Greene, 2005) was embedding digitally recorded spoken words in white noise (Experiment 1 ). The auditory analog to fragmenting typewritten words (e.g., Cleary \& Greene, 2000; Peynircioğlu, 1990) was fragmenting digitally recorded spoken words through phoneme splicing (e.g., Warren \& Warren, 1970).

\section{EXPERIMENT 1}

The purpose of Experiment 1 was to determine whether an auditory recognition without identification effect could be found. Participants listened to digitally recorded spoken words at study, and were then tested with an auditory recognition test containing studied and unstudied words, each of which was embedded in white noise. For each word presented at test, participants were first asked to identify the word through the white noise. Whether or not identification was successful, participants were then asked to rate the likelihood that the test word was a studied one.

\section{Method}

Participants. Twenty-nine Iowa State University undergraduates participated in exchange for credit toward an introductory course in psychology.

Materials. Materials were 120 words taken from Gibson and Watkins's (1988) pool. The words were two- and three-syllable words (all but one of the 120 words contained three syllables). Each of the 120 words was spoken in a female voice and digitally recorded as a sound file (in the ".wav" format) for use with E-Prime software. In addition to this pool of words, a second pool was created in which each of the digitally recorded sound files was embedded in white noise through the use of sound editing software (Creative Wave Studio and Adobe Audition). The noise-to-sound ratio was adjusted to approximately $5: 1$, in order to produce identification rates comparable to those in prior studies in which auditorily recorded words were embedded in white noise (e.g., Pilotti, Bergman, Gallo, Sommers, \& Roediger, 2000).

For every participant, 60 words from the first pool were randomly chosen by the computer program for auditory presentation at study; these study words were not embedded in white noise. For every participant, all of the 120 white-noise-embedded words from the second pool were used as test stimuli during the test phase. The study and test stimuli were segmented into four study-test blocks. Each block consisted of a 15 -item study list followed by a 30 -item test list. On the test, half of the white-noise-embedded items were words that had been presented at study; half were words that had not been presented at study. Each test list was randomly ordered.

Procedure. All segments of the experiment were conducted on a computer. The procedure was explained to participants before beginning. Each participant completed four blocks; a block consisted of a 15 -word auditorily presented study list followed by a 30 -item test list of white-noise-embedded words. Words were presented during the study phase of a given block through a set of computer speakers, and participants were permitted to adjust the volume via a dial on one of the speakers. Each word was separated by an interstimulus interval 
of $1 \mathrm{sec}$. Participants were instructed to remember each word presented during the study list presentation. The nature of the memory test was also explained to them beforehand. Specifically, they were told that they would be presented with a test list containing words embedded in white noise, half of which would be studied and half unstudied. They were told that they would be asked to identify the words through the white noise and also to make a judgment about whether a given word was presented on the study list.

During the test portion of a given block, the words embedded in white noise were presented through the same speakers as was the study list. For each word presented, participants were first prompted to identify the word embedded in white noise. For this, a dialog box appeared in the center of the screen after the word had been played through the speakers. The dialog box prompted participants to identify the word by typing it in. Participants could respond either by typing in a word and pressing the "Enter" key, or by simply pressing the "Enter" key. After pressing "Enter," they were prompted to type in a recognition rating, indicating how likely they thought it was that the word embedded in white noise had been presented at study. They were instructed to give a rating between 0 and $10(0=$ definitely not studied; $10=$ definitely studied). Participants could only respond by typing in an integer between 0 and 10 and pressing the "Enter" key. If they typed a number larger than 10, or pressed the "Enter" key without typing a number, the question would be repeated. It was emphasized in the instructions that participants should rate every test word, even when the word itself could not be identified through the white noise.

If a participant had successfully identified a test word on the first attempt by typing in the correct word when first asked, after giving a rating he or she was immediately presented with the next whitenoise-embedded test word. If, however, the participant had not typed in the correct word on the first attempt, after giving a rating he or she was given a second chance to type it in; in this case, participants were asked to take a guess, even if they felt that they still could not identify the word that had been presented. If the word typed on the second chance trial happened to be the correct one, the rating given to that item was classified as corresponding to an identified item. This was done to ensure that any findings would not be attributable to participants withholding responses when asked to identify the words through the white noise on the first attempt, or to participants suddenly thinking of the answer after moving on to the rating. To ensure that any findings would not be attributable to misspellings on the part of participants, the data were checked for spelling errors; those typed responses that were correct, but happened to be spelled incorrectly, were classified as correctly identified, and the ratings given to those items were classified as corresponding to identified items.

\section{Results and Discussion}

Because the interest was in recognition ratings given to words that could not be identified through white noise, it is important first to consider the identification rates themselves. (These identification rates are presented in Table 1.) As can be seen, auditory priming was shown. Participants identified significantly more studied than unstudied words $[t(28)=12.05, S E=.02, p<.001]$. This replicates prior findings of auditory priming (Pilotti et al., 2000).

Of primary interest to the present study are the recognition ratings given to unidentified test items. The recognition ratings presented in Table 2 show that participants gave higher recognition ratings to unidentified studied items than they did to unidentified unstudied items $[t(28)=5.04, S E=.22, p<.001]$. Hence, an auditory recognition without identification effect was shown. This effect was present when items were treated as participants as well, since the mean rating across items was 3.96 for unidentified studied items, and 2.79 for unidentified unstudied items $\left[t_{\text {items }}(103)=5.19, S E=.23, p<.001\right]{ }^{1,2}$

The more peripheral aspects of the data replicate those from prior studies of the recognition without identification phenomenon (e.g., Cleary \& Greene, 2000, 2004). Namely, a $2 \times 2$ identification status (identified vs. unidentified) $\times$ study status (studied vs. unstudied) repeated measures ANOVA performed on recognition ratings revealed a main effect of identification status $[F(1,28)=$ $\left.113.26, M S_{\mathrm{e}}=1.12, p<.001\right]$. As can be seen in Table 2, the main effect was that participants tended to give higher recognition ratings to test items that could be identified through the white noise than to those that could not. As in previous studies of recognition without identification, this likely reflects an attribution of identifiability to study status (e.g., Cleary \& Greene, 2000). Not surprisingly, a main effect of study status also emerged $[F(1,28)=$ 198.17, $\left.M S_{\mathrm{e}}=1.29, p<.001\right]$. In addition, a significant interaction emerged: The level of old-new discrimination was greater for identified than for unidentified test items $\left[F(1,28)=76.54, M S_{\mathrm{e}}=1.30, p<.001\right]$. Both of these main effects, and the interaction, replicate prior patterns shown in studies of recognition without identification. Therefore, these patterns will not be discussed further.

\section{EXPERIMENT 2}

Experiment 1 demonstrated that an auditory form of recognition without identification can be found. The purposes of Experiment 2 were to (1) extend the generality of the auditory recognition without identification effect reported in Experiment 1 and (2) investigate what features might be used to give rise to spoken word recognition in the absence of identification. Participants might be able to recognize phoneme information as having been heard at study, even when that phoneme information cannot be used to identify the words.

Table 1

Mean Identification Rates for Studied Versus Unstudied Items in Experiments 1-3B

\begin{tabular}{|c|c|c|c|c|c|c|}
\hline \multirow[b]{2}{*}{ Experiment } & \multirow[b]{2}{*}{ Study List } & \multirow[b]{2}{*}{ Test List } & \multicolumn{2}{|c|}{ Studied } & \multicolumn{2}{|c|}{ Unstudied } \\
\hline & & & $M$ & $S D$ & $M$ & $S D$ \\
\hline 1 & Auditory (female) & White noise & .63 & .10 & .39 & .09 \\
\hline 2 & Auditory (female) & Fragment & .65 & .19 & .34 & .14 \\
\hline $3 \mathrm{~A}$ & Auditory (male) & White noise & .58 & .13 & .35 & .09 \\
\hline 3B & Visual & White noise & .47 & .14 & .30 & .11 \\
\hline
\end{tabular}

Note-Test voice was always female. 
Table 2

Mean Recognition Ratings As a Function of Study Status and

Identification Status in Experiments 1-3B

\begin{tabular}{|c|c|c|c|c|c|c|c|c|c|c|}
\hline \multirow[b]{3}{*}{ Experiment } & \multirow[b]{3}{*}{ Study List } & \multirow[b]{3}{*}{ Test List } & \multicolumn{4}{|c|}{ Unidentified } & \multicolumn{4}{|c|}{ Identified } \\
\hline & & & \multicolumn{2}{|c|}{ Studied } & \multicolumn{2}{|c|}{ Unstudied } & \multicolumn{2}{|c|}{ Studied } & \multicolumn{2}{|c|}{ Unstudied } \\
\hline & & & $M$ & $S D$ & $M$ & $S D$ & $M$ & $S D$ & $M$ & $S D$ \\
\hline 1 & Auditory (female) & White noise & 3.95 & 1.48 & 2.84 & 1.58 & 7.90 & 1.13 & 3.08 & 1.58 \\
\hline 2 & Auditory (female) & Fragment & 3.38 & 1.85 & 2.58 & 1.35 & 8.52 & 0.87 & 3.13 & 1.97 \\
\hline $3 \mathrm{~A}$ & Auditory (male) & White noise & 3.65 & 1.38 & 3.22 & 1.40 & 8.03 & 1.01 & 3.96 & 1.94 \\
\hline $3 \mathrm{~B}$ & Visual & White noise & 2.48 & 1.27 & 2.28 & 1.36 & 7.27 & 1.57 & 3.19 & 2.07 \\
\hline
\end{tabular}

Note-Test voice was always female.

This possibility was examined in Experiment 2 . Rather than preventing identification through embedding spoken words in white noise, identification was hindered through fragmentation of the digitally recorded words that spliced particular phonemes out of each spoken word recording. Such phoneme splicing has been used in the past to study such phonological effects as the phoneme restoration effect (e.g., Warren \& Warren, 1970).

By isolating phoneme information at the time of test, Experiment 2 also served the purpose of determining whether phonemes are a type of isolable item feature that can be used in the episodic recognition of spoken words. As mentioned, the recognition without identification paradigm can be a useful means of probing the features used in recognition memory. Experiment 2 extended this use to the auditory modality.

\section{Method}

Participants. Twenty-six Iowa State University undergraduates participated in exchange for credit toward their introductory psychology classes.

Materials. The materials were the same as those used in Experiment 1, except that the pool of 120 test items were fragmented forms of the words from the pool of 120 possible study items: Specifically, the 120 female voice recordings from the study pool of Experiment 1 were fragmented by splicing particular phonemes from these recordings. The splicing of phonemes was done using Adobe Audition. Approximately four phonemes per word were left intact, and the first phoneme was included in each auditory fragment.

Procedure. The procedure was the same as that used in Experiment 1 , except that, rather than hearing the test words embedded in white noise, participants heard fragmented forms of words, half of which corresponded to words heard at study and half to new, unstudied words.

\section{Results and Discussion}

The mean identification rates are presented in Table 1. As in Experiment 1, auditory priming was shown. Participants identified significantly more studied than unstudied words $[t(25)=13.04, S E=.02, p<.001]$.

Of primary interest to the present study are the recognition ratings given to unidentified test items. The recognition ratings are presented in Table 2 . As can be seen in Table 2, participants gave higher recognition ratings to unidentified studied items than to unidentified unstudied items $[t(25)=3.23, S E=.25, p<.01]$. Given that identification was hindered through the isolation of particular word phonemes, this finding suggests that phonemes are a type of isolable feature that can give rise to recognition without identification of spoken words.

\section{EXPERIMENT 3A}

Experiments 1 and 2 demonstrated an auditory recognition without identification effect; the recognition without identification phenomenon is not, therefore, limited to situations in which the unidentifiable test items are visual in nature. Experiment 2 further showed that spoken word recognition without identification can be based on phoneme information. Even if the auditory recognition without identification effect shown in Experiment 1 was based on phoneme information, that information could be perceptually specific (i.e., it could require a reinstatement of specific auditory features from study to test), or it could be information abstracted away from particular studied episodes. If the latter, the effect would involve a role of preexisting knowledge.

The recognition without identification effect found with visual word fragments has been shown to persist when such surface features as the lettering (capital vs. lowercase) or the presentation modality (auditory vs. visual) have changed from study to test (Cleary \& Greene, 2000). Thus, visual forms of recognition without identification do not appear to be dependent on the presence of a perceptual match from study to test. Experiments $3 \mathrm{~A}$ and $3 \mathrm{~B}$ of the present study examined whether the auditory recognition without identification effect would be perceptually specific, or whether it would persist when the perceptual specifics changed from study to test. In Experiment 3A, the voice was changed from study to test. The study items were presented in a male voice, and the test items were presented in a female voice. In Experiment 3B, the modality was changed from study to test. The study items were presented visually, but the test list was presented auditorily.

\section{Method}

Participants. Fifty-seven Iowa State University undergraduates participated in exchange for credit toward their introductory psychology classes.

Materials. The materials were the same as those used in Experiment 1 , except that the study items were selected from a pool of 120 digitally recorded words spoken in a male voice, rather than from the pool recorded in a female voice for Experiment 1.

Procedure. The procedure was the same as that used in Experiment 1.

\section{Results and Discussion}

The mean identification rates are presented in Table 1. As in Experiment 1, auditory priming was shown. Participants identified significantly more studied than unstudied words $[t(56)=17.54, S E=.01, p<.001]$. 
Of primary interest to the present study are the recognition ratings given to unidentified test items. The recognition ratings presented in Table 2 show that participants gave higher recognition ratings to unidentified studied items than to unidentified unstudied items $[t(56)=4.39$, $S E=.10, p<.001]$, even when the voice was changed from male to female from study to test. Hence, auditory recognition without identification behaves in much the same way as does visual recognition without identification (e.g., Cleary \& Greene, 2000), in that it persists when the surface features have been changed from study to test.

\section{EXPERIMENT 3B}

Experiment 3B involved a stronger means of changing the perceptual specifics of the stimulus items from study to test. Specifically, Experiment 3B examined the effect of changing the presentation modality from study to test on the auditory recognition without identification effect. The study list was presented visually and the test list was the same as in Experiments 1 and 3A.

\section{Method}

Participants. Forty-eight Iowa State University undergraduates participated in exchange for credit toward their introductory psychology classes.

Materials. The materials were the same as those used in Experiment 1 , except that the study items were presented visually instead of auditorily. Each typewritten word from the pool of 120 possible study items was saved in a text file for use with E-Prime software.

Procedure. The procedure was the same as that used in Experiment 1 , with the exception that the study list was presented visually. Each study word appeared in the top left corner of the computer screen for $2 \mathrm{sec}$, and each word was separated by an interstimulus interval of $1 \mathrm{sec}$.

\section{Results and Discussion}

The mean identification rates are presented in Table 1. As in Experiments 1, 2, and 3A, auditory priming was shown. Participants identified significantly more studied than unstudied words $[t(47)=12.80, S E=.01, p<$ $.001]$.

Of primary interest to the present study are the recognition ratings given to unidentified test items. The recognition ratings are presented in Table 2. As can be seen in Table 2, participants gave higher recognition ratings to unidentified studied items than to unidentified unstudied items $[t(47)=2.35, S E=.08, p<.05]$, even when the presentation modality was changed from visual to auditory at test. As in Experiment 3A, auditory recognition without identification persisted when the perceptual features were changed from study to test.

\section{EXPERIMENT 4}

Although the auditory recognition without identification effect appears to persist when such perceptual specifics as the voice and the presentation modality are changed from study to test, a look at Table 2 suggests that the effect found in Experiments 3A and 3B might have been smaller than the effect found in Experiment 1. Furthermore, a look at Table 2 also suggests that ratings may have been lower overall in the modality mismatch condition of Experiment 3B than in the prior experiments. To examine these possibilities, Experiment 4 directly compared the auditory recognition without identification effect across matching and mismatching study-test modalities in a within-experiment comparison. In addition, because it is conceivable that visual or auditory study presentation itself might lead to a larger recognition without identification effect, we examined matching and mismatching study-test modalities using both auditory and visual testing situations. Doing so would allow us to tease apart the effects of study modality, test modality, and study-test match on the recognition without identification effect.

\section{Method}

Participants. Participants were 220 Colorado State University undergraduates, who participated in exchange for credit toward their introductory psychology classes.

Materials. The materials were the same as those used in Experiments 1 and 3B, except that, in addition to auditory and visual study conditions, both auditory and visual test conditions were used. For the visual test condition, the words were rapidly flashed and masked with a 100 -msec mask.

Procedure. The procedure was the same as that used in Experiments 1 and 3B, except that participants were randomly assigned to either the auditory-auditory condition (auditory study and auditory test lists), the visual-auditory condition (visual study and auditory test lists), the visual-visual condition (visual study and visual test lists) or the auditory-visual condition (auditory study and visual test lists). For the visual test condition, each test word was rapidly flashed and was preceded and followed by a mask of dollar signs. The duration of each word was set at $30 \mathrm{msec}$ in E-Prime at a monitor refresh rate of $85 \mathrm{~Hz}$, with a $40-\mathrm{msec}$ prerelease setting. Each mask was set at $100 \mathrm{msec}$ with a $40-\mathrm{msec}$ prerelease setting. As in the auditory test condition, participants were first prompted to identify the word; they were then prompted to rate the likelihood that the word had appeared at study. Following the rating, if a participant had not identified the word on the first attempt, he or she was given a second chance to do so and was encouraged to guess on the second attempt.

\section{Results and Discussion}

Identification rates. The mean identification rates are presented in Table 3 . Repetition priming was shown. Overall, a $2 \times 2 \times 2$ study status (studied vs. unstudied) $\times$ study modality (visual vs. auditory) $\times$ test modality (visual vs. auditory) mixed ANOVA revealed a significant main effect of study status on the proportion of test items identified $\left[F(1,216)=872.81, M S_{\mathrm{e}}=.007, p<.001\right]$. The magnitude of this repetition priming effect was affected by study-test match, as revealed by a significant three-way interaction $\left[F(1,216)=83.29, M S_{\mathrm{e}}=.007\right.$, $p<.001]$. In replication of prior findings in the literature (e.g., Pilotti et al., 2000; Roediger \& Blaxton, 1987), more repetition priming occurred when there was a modality match than when there was a modality mismatch from study to test. As can also be seen in Table 3, more priming occurred in the auditory test modality than in the visual test modality; this was shown by a marginally significant two-way study status $\times$ test modality interaction $\left[F(1,216)=3.49, M S_{\mathrm{e}}=.007, p=.06\right]$. There was a main effect of test modality on identification rates $[F(1,216)=$ $\left.13.68, M S_{\mathrm{e}}=.04, p<.001\right]$, such that more items were identified overall in the auditory test modality than in the 
Table 3

Mean Identification Rates Across Modality Conditions

in Experiment 4

\begin{tabular}{|c|c|c|c|c|c|c|c|c|}
\hline \multirow[b]{4}{*}{ Study Modality } & \multicolumn{8}{|c|}{ Test Modality } \\
\hline & \multicolumn{4}{|c|}{ Auditory } & \multicolumn{4}{|c|}{ Visual } \\
\hline & \multicolumn{2}{|c|}{ Studied } & \multicolumn{2}{|c|}{ Unstudied } & \multicolumn{2}{|c|}{ Studied } & \multicolumn{2}{|c|}{ Unstudied } \\
\hline & $M$ & $S D$ & $M$ & $S D$ & $M$ & $S D$ & $M$ & $S D$ \\
\hline Auditory & .57 & .10 & .26 & .07 & .34 & .22 & .20 & .17 \\
\hline Visual & .40 & .11 & .23 & .07 & .45 & .26 & .17 & .15 \\
\hline
\end{tabular}

visual test modality. There was no significant study status $\times$ study modality interaction $\left[F(1,216)=0.09, M S_{\mathrm{e}}=\right.$ $.007, p=.77]$, indicating that the study modality did not significantly affect the repetition priming effect, which appears to have been affected only by the test modality.

\section{Recognition Ratings Given to Unidentified Test Items: Recognition Without Identification}

The recognition without identification effect. Of primary interest to the present study are the recognition ratings given to unidentified test items. The recognition ratings in Table 4 show that several significant patterns emerged in Experiment 4. First, a $2 \times 2 \times 2$ study status (studied vs. unstudied) $\times$ study modality (visual vs. auditory) $\times$ test modality (visual vs. auditory) mixed ANOVA performed on ratings given to unidentified test items revealed a main effect of study status $[F(1,216)=$ $\left.29.82, M S_{\mathrm{e}}=.32, p<.001\right]$, such that participants gave higher ratings overall to unidentified studied items than to unidentified unstudied items. That is, the recognition without identification effect occurred, and, as revealed by a main effect of study status emerging from a $2 \times 2$ study status $X$ study modality mixed ANOVA performed separately for each test modality, it occurred in both the auditory $\left[F(1,108)=29.20, M S_{\mathrm{e}}=.30, p<.001\right]$ and visual $\left[F(1,108)=5.76, M S_{\mathrm{e}}=.34, p<.02\right]$ test modalities.

The effect of study modality on recognition without identification. The second main pattern to emerge from the $2 \times 2 \times 2$ ANOVA was that study modality did not significantly impact the magnitude of the recognition without identification effect, as revealed by a nonsignificant study status $\times$ study modality interaction $[F(1,216)=$ $\left.0.12, M S_{\mathrm{e}}=.32, p=.65\right]$. Therefore, it does not appear that the particular study modality (auditory or visual) has any bearing on the magnitude of the recognition without identification effect obtained at test.

The effect of test modality on recognition without identification. However, the third pattern to emerge from this analysis was that the test modality had an impact on the magnitude of the recognition without identification effect, as revealed by a significant study status $\times$ test modality interaction $\left[F(1,216)=3.93, M S_{\mathrm{e}}=.32, p<.05\right]$. This interaction was such that the magnitude of the recognition without identification effect was greater in the auditory testing situation than in the visual testing situation. This interaction should be interpreted with caution, however, as it does not necessarily suggest that the recognition without identification effect itself will always be larger in the auditory test modality than in the visual test modality. The reason is that it is difficult to equate different test modalities on the amount of featural information that can be extracted from a degraded presentation. It may be that more word features can be extracted from a spoken word embedded in white noise than from a briefly flashed visual word that is preceded and followed by a mask.

In support of the idea that more information may have been available in the white-noise-embedded auditory presentations than in the rapidly flashed, masked visual presentations, identification rates overall were higher in the auditory testing situation than in the visual testing situation. With a longer presentation duration at test, the magnitude of the visual recognition without identification effect might possibly have been larger; in fact, previous data from our lab suggest that as the presentation duration of the rapidly flashed test items increases, so does the magnitude of the recognition without identification effect (Morris, Cleary, \& Still, 2007). Moreover, there is some evidence to suggest that reducing the number of features present in the test item-for example, by reducing the number of letters in a fragment from four to two-reduces the magnitude of the recognition without identification effect (Cleary \& Greene, 2000).

Still, it may be difficult to vary the sound-to-noise ratio of the auditory presentation and the duration of the visual flash so that one can be certain that feature extraction is equated in both cases. Even attempting to equate auditory

Table 4

Ratings Given to Unidentified Items Across Varying Study and Test Modalities

Test Modality

\begin{tabular}{|c|c|c|c|c|c|c|c|c|}
\hline \multirow[b]{3}{*}{ Study Modality } & \multicolumn{4}{|c|}{ Auditory } & \multicolumn{4}{|c|}{ Visual } \\
\hline & \multicolumn{2}{|c|}{ Studied } & \multicolumn{2}{|c|}{ Unstudied } & \multicolumn{2}{|c|}{ Studied } & \multicolumn{2}{|c|}{ Unstudied } \\
\hline & $M$ & $S D$ & $M$ & $S D$ & $M$ & $S D$ & $M$ & $S D$ \\
\hline Auditory & 3.33 & 1.17 & 2.83 & 1.31 & 3.25 & 1.48 & 3.11 & 1.52 \\
\hline Visual & 2.68 & 1.36 & 2.37 & 1.28 & 3.71 & 1.56 & 3.48 & 1.53 \\
\hline
\end{tabular}


and visual test modalities in terms of identification rates would not necessarily mean that the test modalities had been equated in terms of the number or quality of the features that could be extracted in the absence of identification. One would be left to draw inferences, in a somewhat circular reasoning process, about whether the two had been equated in terms of feature extraction from the magnitude of the recognition without identification effect itself. In short, the fact that the recognition without identification effect is shown to be smaller here in the visual than in the auditory test modality should be interpreted with caution.

The effect of study-test match on recognition without identification. The lack of a study modality main effect suggests that study modality does not affect the recognition without identification effect. The trend present in the data would suggest that, if anything, the study-test match is more likely than study modality to affect the recognition without identification effect. However, no significant effects of study-test match on the magnitude of the recognition without identification effect were found in the present study: The fourth main pattern to emerge from the $2 \times 2 \times 2$ ANOVA was that there was no significant overall effect of study-test match on the magnitude of the recognition without identification effect (as revealed by a nonsignificant study status $\times$ study modality $\times$ test modality interaction) $\left[F(1,216)=1.66, M S_{\mathrm{e}}=.32, p=.20\right]$. To follow up, a $2 \times 2$ study status $\times$ study modality ANOVA was performed separately for ratings given to unidentified items in each test modality. There was no significant twoway interaction revealed for either the visual test modality $\left[F(1,108)=0.33, M S_{\mathrm{e}}=.34, p=.57\right]$ or for the auditory test modality $\left[F(1,108)=1.61, M S_{\mathrm{e}}=.30, p=.21\right]$. Thus, study-test match appears to have a negligible impact on the magnitude of the recognition without identification effect reported here. This suggests, with particular relevance to the present study, that the information driving the auditory recognition without identification effect reported here may be information abstracted away from the perceptual specifics of the studied episodes.

\section{The Effect of Modality on Overall Recognition Ratings to Unidentified Items}

Other findings that emerged from the $2 \times 2 \times 2$ ANOVA are the following: First, while there was no main effect of study modality on ratings given to unidentified items $\left[F(1,216)=0.14, M S_{\mathrm{e}}=3.64, p=.71\right]$, there was a significant main effect of test modality on these ratings $\left[F(1,216)=10.31, M S_{\mathrm{e}}=3.64, p<.01\right]$. As shown in Table 4, this effect was such that participants gave higher ratings overall in the visual test modality than in the auditory test modality. This pattern is interesting in light of the fact that the opposite pattern was shown for repetition priming: More test items were identified in the auditory test modality than in the visual test modality. In cases in which more test items can be identified, participants might tend to attribute an inability to identify an item to the likelihood that it was not studied. The same argument was made by Cleary et al. (2004) regarding a similar pattern shown with recognition ratings given to picture fragments that were easier, not more difficult, to identify; picture fragments of a class that were easier to identify (geon fragments) tended to receive lower ratings overall than picture fragments of a class that were more difficult to identify (nongeon fragments).

\section{The Effect of Study-Test Match on Overall Recognition Ratings to Unidentified Items}

Finally, a significant study modality $\times$ test modality interaction emerged $\left[F(1,216)=7.04, M S_{\mathrm{e}}=3.64, p<\right.$ $.01]$, such that participants gave higher ratings overall in cases in which there was a modality match from study to test than they did in cases in which there was a modality mismatch from study to test. This result replicates and extends findings reported by Westerman, Lloyd, and Miller (2002). Westerman et al. showed that when there is a perceived study-test match, such as when a counterfeit study list is presented in the same modality as the recognition test list, participants attribute that perceived match to the study status of a test item. We obtained a similar phenomenon in the present study. When participants could not identify a recognition test item, they found it more likely that the item was studied when the test modality matched the study modality than when it did not.

\section{GENERAL DISCUSSION}

The present findings extend the recognition without identification effect (e.g., Cleary \& Greene, 2000; Peynircioğlu, 1990) to the auditory modality. Auditory identification was prevented at test by embedding spoken words in white noise and by fragmenting spoken words through the splicing of phonemes from these words. The experiments reported here consistently demonstrated that when identification of spoken words is prevented, episodic recognition can still occur.

In each block of each experiment reported here, participants were first presented a study list followed by an auditory test list (with the exception of the visual test condition of Experiment 4). For each auditory test item, participants first attempted to identify the word. In Experiments 1, 3A, and $3 \mathrm{~B}$, participants attempted to identify spoken words that were embedded in white noise. In Experiment 2, participants attempted to identify fragmented spoken words. After attempting to identify a given spoken word, participants were asked to rate the likelihood that it was studied, whether or not the word could be identified. In Experiment 4 , the effects of modality match from study to test were examined. All five experiments presented here show that participants' ratings discriminated between studied and unstudied items among spoken words that went unidentified at test. In short, auditory recognition without identification was shown.

Experiments $3 \mathrm{~A}$ and $3 \mathrm{~B}$ suggest that the auditory recognition without identification effect reported here is not dependent on a reinstatement of the perceptual specifics of the studied episodes at test; that is, the effect is not dependent on a perceptual match from study to test. Past studies have shown that visual forms of recognition without identification, such as the effect found with visual word fragments (Cleary \& Greene, 2000) and the effect found 
with rapid, masked visual presentation (Cleary \& Greene, 2004), still occur when such perceptual specifics as the lettering (capital vs. lowercase) and the presentation modality (visual vs. auditory study lists) have changed from study to test. The present study shows the same to be true of auditory recognition without identification. The effect still occurs when such perceptual specifics as the voice (male vs. female) and the presentation modality (visual vs. auditory study list) have been changed from study to test.

Some cross-experimental comparisons with visual recognition without identification have shown the effect to diminish (although still be present) with a change in such perceptual specifics as the presentation modality (Cleary \& Greene, 2000, 2004); however, Experiment 4 of the present study found no significant effects of study-test match on the recognition without identification effect. It is possible that if we were to increase the magnitude of the visual recognition without identification effect-for example, by increasing the presentation duration of the visually presented test items - a significant impact of studytest match would begin to emerge in that condition. Still, as is consistent with other findings in the literature, the recognition without identification effect itself is generally not dependent on a perceptual match from study to test; it generally occurs even when the perceptual specifics have changed from study to test. The effect, particularly the auditory recognition without identification effect reported here, likely involves a form of information abstracted away from the specifics of study presentation.

What kind of auditory information might be abstracted away from a spoken word for later recognition? Cleary (2002) suggested that, because the effect persists when the presentation modality has changed from study to test, the recognition without identification effect found with visual word fragments might involve preexisting knowledge about word orthography in cases in which the information used to recognize appears to have been abstracted away from the specifics of study presentation. It is possible that the auditory recognition without identification effect reported here involves preexisting knowledge about word phonology, and that this component allows the effect to persist when the perceptual specifics have changed from study to test.

The paradigm used to elicit recognition without identification has been used in the past as a means of identifying the kinds of isolable features that can give rise to recognition memory in standard list-learning paradigms (e.g., Cleary, 2004; Cleary et al., 2004). A basic assumption of many memory researchers is that memory traces for studied events exist as compilations of separable features (Tulving \& Bower, 1974). Although many formal models of memory are based on this premise (e.g., Clark \& Gronlund, 1996; Hintzman, 1988; Nairne, 1990, 2001), little is known about what specific types of item features can actually be used in episodic recognition, when presented in isolation. Thus, as a means to empirically validate the long-held pretheoretical assumption about the use of features in recognition memory, it is important to (1) demonstrate that particular kinds of isolated item features can produce episodic recognition and (2) systematically iden- tify the specific kinds of isolated item features that can produce recognition.

The auditory recognition without identification paradigm used in the present study presents a potential means of identifying the kinds of auditory features that can give rise to recognition of auditory stimuli. As illustrated in Experiment 2, the paradigm can be used to isolate particular kinds of auditory features for examination in recognition. In Experiment 2, particular word phonemes were isolated at test by splicing some phonemes from the digital recordings of spoken words, leaving only a fraction of each word's phonemes available in the auditory test fragments. The fact that auditory recognition without identification can be based on isolated sets of word phonemes suggests that the phoneme may be a type of word feature that is stored in memory when a word is spoken. Thus, when no other information is available at test, it is possible that episodic recognition can be based on the stored phonemic information. This is consistent with other findings in the literature that have suggested that a spoken word's phonological characteristics can become activated at encoding, and can thus make phonologically similar lures seem familiar on a later recognition test (e.g., Wallace, Shaffer, Amberg, \& Silvers, 2001). However, the present findings are also quite distinctive in showing familiarity with phonological information that has been isolated from particular words.

Given that the recognition without identification effect can be extended to the auditory modality, the paradigm used to elicit auditory recognition without identification may be useful for systematically identifying other types of auditory features used in recognition. For example, can auditory recognition without identification be shown for sentence stimuli, or for musical stimuli that are embedded in white noise? If so, it may be possible to isolate the featural components of these types of stimuli to investigate their role in recognition.

\section{AUTHOR NOTE}

Some of the data reported here were collected as part of M.M.W.'s undergraduate research project for the Ronald E. McNair Scholar Program at Iowa State University under the guidance of A.M.C. The research reported here was funded by National Science Foundation Grants 0349088 and 0638486 to A.M.C. The authors thank Jessica Beyer and Holly Owen for assistance with data collection at Colorado State University. Correspondence concerning this article should be addressed to A. M. Cleary, Department of Psychology, Colorado State University, 1876 Campus Delivery, Fort Collins, CO 80523 (e-mail: anne.cleary@colostate.edu).

\section{REFERENCES}

Biederman, I. (1987). Recognition-by-components: A theory of human image understanding. Psychological Review, 94, 115-147.

Clark, S. E., \& Gronlund, S. D. (1996). Global matching models of recognition memory: How the models match the data. Psychonomic Bulletin \& Review, 3, 37-60.

Cleary, A. M. (2002). Recognition with and without identification: Dissociative effects of meaningful encoding. Memory \& Cognition, 30, 758-767.

Cleary, A. M. (2004). Orthography, phonology, and meaning: Word features that give rise to feelings of familiarity in recognition. Psychonomic Bulletin \& Review, 11, 446-451.

Cleary, A. M., \& Greene, R. L. (2000). Recognition without identification. Journal of Experimental Psychology: Learning, Memory, \& Cognition, 26, 1063-1069. 
Cleary, A. M., \& Greene, R. L. (2001). Memory for unidentified items: Evidence for the use of letter information in familiarity processes. Memory \& Cognition, 29, 540-545.

Cleary, A. M., \& Greene, R. L. (2004). True and false memory in the absence of perceptual identification. Memory, 12, 231-236.

Cleary, A. M., \& Greene, R. L. (2005). Recognition without perceptual identification: A measure of familiarity? Quarterly Journal of Experimental Psychology, 58A, 1143-1152.

Cleary, A. M., Langley, M. M., \& Seiler, K. R. (2004). Recognition without picture identification: Geons as components of the pictorial memory trace. Psychonomic Bulletin \& Review, 11, 903-908.

Gibson, J. M., \& WatKins, M. J. (1988). A pool of 1,086 words with unique two-letter fragments. Behavior Research Methods, Instruments, \& Computers, 20, 390-397.

Hintzman, D. L. (1988). Judgments of frequency and recognition memory in a multiple-trace memory model. Psychological Review, 95, 528-551.

Lloyd, M. E., Westerman, D. L., \& Miller, J. M. (2007). Familiarity from orthographic information: Extensions of the recognition without identification effect. Memory \& Cognition, 35, 107-112.

Morris, A. L., Cleary, A. M., \& Still, M. L. (2007). Electrodermal recognition without identification: When skin conductance responses show recognition of unidentified items. Manuscript submitted for publication.

NAIRne, J. S. (1990). A feature model of immediate memory. Memory \& Cognition, 18, 251-269.

NAIRne, J. S. (2001). A functional analysis of primary memory. In H. L. Roediger III, J. S. Nairne, I. Neath, \& A. M. Surprenant (Eds.), The nature of remembering (pp. 283-296). Washington, DC: American Psychological Association.

PeynircioĞLU, Z. F. (1990). A feeling-of-recognition without identification. Journal of Memory \& Language, 29, 493-500.
Pilotti, M., Bergman, E. T., Gallo, D. A., Sommers, M., \& RoediGER, H. L., III (2000). Direct comparison of auditory implicit tests. Psychonomic Bulletin \& Review, 7, 347-353.

Roediger, H. L., III, \& Blaxton, T. A. (1987). Effects of varying modality, surface features, and retention interval on priming in wordfragment completion. Memory \& Cognition, 15, 379-388.

Tulving, E., \& Bower, G. H. (1974). The logic of memory representations. In G. H. Bower (Ed.), The psychology of learning and motivation (Vol. 8, pp. 265-300). New York: Academic Press.

Wallace, W. P., Shaffer, T. R., Amberg, M. D., \& Silvers, V. L. (2001). Divided attention and prerecognition processing of spoken words and nonwords. Memory \& Cognition, 29, 1102-1110.

WARren, R. M., \& WARrEn, R. P. (1970). Auditory illusions and confusions. Scientific American, 223, 30-36.

Westerman, D. L., Lloyd, M. L., \& Miller, J. M. (2002). The attribution of perceptual fluency in recognition memory: The role of expectation. Journal of Memory \& Language, 47, 607-617.

\section{NOTES}

1. The degrees of freedom reflect the fact that some items were identified $100 \%$ of the time, particularly in the studied condition. These items could not be used in the items analysis, as the interest was in recognition ratings given to those items that went unidentified.

2 . This auditory recognition without identification effect was significant at the .01 level when items were treated as participants in all subsequent experiments and conditions except for Experiment $3 \mathrm{~B}$, in which $p$ was .177 when items were treated as participants.

(Manuscript received March 26, 2006; revision accepted for publication March 9, 2007.) 